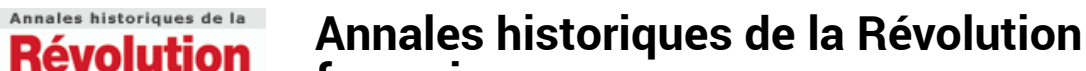

française française

\section{8 | avril-juin 2002}

La Révolution et le Droit

\section{Table ronde}

La Révolution française et la bourgeoisie

Andreï Tyrsenko

\section{(2) OpenEdition}

Journals

Édition électronique

URL : https://journals.openedition.org/ahrf/2253

DOI : 10.4000/ahrf.2253

ISSN : 1952-403X

Éditeur :

Armand Colin, Société des études robespierristes

Édition imprimée

Date de publication : 1 juin 2002

Pagination : 200-202

ISSN : 0003-4436

Référence électronique

Andreï Tyrsenko, «Table ronde », Annales historiques de la Révolution française [En ligne], 328 | avril-juin 2002, mis en ligne le 11 mai 2006, consulté le 24 avril 2022. URL : http://journals.openedition.org/ahrf/ 2253 ; DOI : https://doi.org/10.4000/ahrf.2253

Ce document a été généré automatiquement le 24 avril 2022.

Tous droits réservés 


\title{
Table ronde
}

\author{
La Révolution française et la bourgeoisie ${ }^{1}$
}

\section{Andreï Tyrsenko}

1 Le 24 avril 2001, à l'Institut de l'histoire universelle, s'est tenue une table ronde organisée par le Centre d'études françaises et par le Conseil scientifique d'histoire des mouvements sociaux.

2 Les interventions à la table ronde se sont articulées autour de l'exposé, présenté par A. Tchoudinov. Dans son exposé A. Tchoudinov a proposé d'échanger des points de vue sur la catégorie de "bourgeoisie » ainsi que sur le rôle de ce groupe social dans la Révolution. Pour l'historiographie russe ce sujet requiert l'attention soutenue des spécialistes compte tenu de la thèse du "caractère bourgeois " de la Révolution française et du rôle primordial de la bourgeoisie dans la Révolution, deux notions de base pour l'historiographie soviétique. Partant de l'assertion que l'historiographie soviétique ne séparait pas la «bourgeoisie » comme concept marxiste signifiant une classe de la société capitaliste de la notion de bourgeoisie courante dans le discours du XVIII ${ }^{\mathrm{e}}$ siècle.

Se référant aux recherches françaises récentes, Tchoudinov souligne que même l'historiographie classique a fini par accepter de parler des «bourgeoisies» pour indiquer l'hétérogénéité de cette catégorie sociale. Après s'être attaché à la question de savoir laquelle des bourgeoisies a réalisé la Révolution, Tchoudinov a abouti à conclure que la Révolution ne peut pas être qualifiée d'affaire de la bourgeoisie, ni même de l'une des bourgeoisies. C'est pourquoi il propose d'employer la notion de bourgeoisie au sens historique du mot, comme concept culturel et juridique, afin d'éviter les confusions de sens. En effet, insiste-t-il, la Révolution n'a jamais été fonction de la bourgeoisie capitaliste au sens marxiste du terme, celle-ci ne jouant pas un rôle politique important à l'époque. De plus, les effets à long terme de la Révolution, que Tchoudinov estime néfastes pour les perspectives de l'économie capitaliste, n'ont pas profité ipso facto à ce groupe social. Tchoudinov appelle à élaborer un nouveau paradigme de la Révolution, étant donné l'impossibilité où l'on est d'adhérer au paradigme de la "Révolution bourgeoise ", dépourvu de contenu épistémologique à la lumière d'études récentes. 
4 L'exposé de Tchoudinov a été suivi d'une discussion. Le débat a été engagé par A. Gordon. D'après lui il est fort louable de chercher à repenser le rôle de la bourgeoisie dans la Révolution ainsi que d'avancer un nouveau paradigme de la Révolution. D'une part, Gordone souhaite débarrasser le concept de Révolution française de sa rationalité transitologique simpliste. D'autre part, il tient à le remplacer par le concept de progrès sous son aspect subjectif et de valeur. C'est sur cette voie qu'on peut représenter la Révolution par l'Événement, unique dans l'Histoire, par l'être particulier du sujet historique. Mais cette transformation épistémologique ne conduit pas forcément à sous-estimer les concepts de longue durée, de cycles macro-économiques, de mentalités qui restent nécessaires à leurs propres domaines. En même temps, les catégories subjectives tels que tempéraments, représentations sociales, etc., permettent de mieux comprendre l'événement historique.

5 V. Smirnov, dans son intervention, a attiré l'attention de l'assistance sur le fait que le concept de «Révolution bourgeoise" fait ressortir le trait important du processus révolutionnaire: la mutation de la bourgeoisie en classe prépondérante de la société. De plus, la Révolution française a été un mouvement national. Si on peut admettre le rôle politique médiocre qui revient à la bourgeoisie industrielle et commerciale dans la Révolution, la participation active de la bourgeoisie de talents aux affaires politiques ne fait aucun doute. V.Smirnov a remarqué aussi que la bourgeoisie a su profiter des effets de la Révolution française à long terme.

6 D.Bovykine a démontré, au sujet de thermidor, que les meneurs de la Révolution de l'époque ne peuvent pas être identifiés à la bourgeoisie, qu'il est erroné de désigner leur politique comme correspondant aux intérêts de la bourgeoisie, et enfin que la Constitution de l'an III cherchait à instaurer plutôt un ordre national qu'un ordre bourgeois.

7 S.Blumenau a mis en garde contre deux écueils à éviter. D'une part, il s'agit de la thèse du rôle dirigeant de la bourgeoisie dans la Révolution, d'autre part, la tendance à sousestimer la position active des entrepreneurs dans l'économie de la fin de l'Ancien Régime et leurs dispositions au changement qui a lieu lors de la Révolution.

8 S.Letchforde a reconnu l'impossibilité où l'on est d'appliquer une analyse macrosociologique aux événements de la Révolution. Il convient pour lui de partager la société révolutionnaire, ce qui ne serait pas dépourvu d'intérêt, en deux camps: le camp révolutionnaire et le camp contre-révolutionnaire et pratiquer, pour ces deux cas, une identification microsociologique. Letchforde adhère au concept de la Révolution bourgeoise, surtout partant du fait que la bourgeoisie a tiré un profit essentiel des transformations révolutionnaires.

9 Z.Tchekanzeva oppose qu'au lieu de reconstruire l'histoire sociopolitique, comme le faisait l'historiographie classique en ayant recours à des définitions précises et à des données quantitatives, il faut repenser l'histoire de la Révolution en s'attachant aux approches qui se sont cristallisées dès les années 1770, surtout dans le cadre du tournant linguistique. Comprendre les groupes sociaux, la bourgeoisie comprise, à l'époque révolutionnaire et repérer ce qui les sépare - mœurs, comportements, coutumes, représentations etc.

10 A.Gladychev a porté son attention sur l'extrapolation de l'expérience historique «bourgeoise» de l'Angleterre des années 1790 ou de la France de l'époque de la Restauration à l'époque révolutionnaire. Il envisage de trouver la solution du problème 
de la bourgeoisie révolutionnaire dans le cadre de la dichotomie entre les représentations sociopolitiques des protagonistes et leurs pratiques réelles. Une telle approche exige de tenir compte de la connexion entre les niveaux micro et macrosociologiques pour construire le social à partir de l'individuel.

NOTES

1.Histoire moderne et contemporaine. 1, 2002. 